\title{
VIEWPOINT
}

\section{Drug eluting stents: maximising benefit and minimising cost}

\section{J Gunn, A C Morton, C Wales, C M H Newman, D C Crossman, D C Cumberland}

Heart 2003;89:127-131

\section{A policy of selective implantation of drug eluting stents, in a minority of lesions most likely to benefit, seems to be a rational way to employ this new and currently costly technology}

See end of article for authors' affiliations

Dr Julian Gunn, Cardiovascular Research Group, Division of Clinical Sciences (Northern General Hospital), University of Sheffield Clinical Sciences Building Northern General Hospital Sheffield, S5 7AU, UK J.Gunn@Sheffield.ac.uk
$T$ he RAVEL and the SIRIUS studies, using a sirolimus eluting stent, report substantial reductions in clinical restenosis with drug eluting compared with bare stents. The drug eluting stent has been hailed as the third great breakthrough in percutaneous coronary intervention (PCI) after the balloon and the stent, but excitement greeting its arrival must be tempered by three facts. Firstly, it is impossible to predict exactly which individual coronary artery stenosis will restenose (making it difficult to know who would benefit from the new stent). Secondly, "real world" PCI includes many lesion types more adverse (and probably more deserving of antirestenosis strategies) than those included in the studies. Thirdly, the first drug eluting stent on the market costs about five times more than a conventional stent (making it economically impossible to treat all lesions). We need to decide how to make rational treatment decisions with this new device within limited health budgets. In this article, we attempt to do exactly that

The first drug eluting stent (Cypher, Cordis) has recently been made commercially available in the UK. Data from the RAVEL study and the "first-in-man" studies suggest that this device, which elutes the drug sirolimus (rapamycin, a macrolide antibiotic, with immunosuppressant, antiproliferative, and antimigratory properties), produces a zero restenosis rate up to, and beyond, one year in selected lesions. ${ }^{1-3}$ Interestingly, the larger SIRIUS study (similar to RAVEL, but performed in the USA), as yet unpublished, has complete follow up data available for the first 400 patients enrolled; the study demonstrates (as its primary end point) a $12 \%$ target vessel failure (TVF) rate in the sirolimus group compared with $23 \%$ in the control group, while the target lesion revascularisation (TLR) rate (an index of which restenotic lesions really matter) was $6.8 \%$ versus $21.5 \%$ - a two thirds reduction. ${ }^{4}$ The growth of in-stent neointima in this study was, as in RAVEL, negligible. The relatively high TVF rate is being explained as angiographic restenosis beyond the ends of the stent, perhaps caused by balloon deployment there. If this simple procedural (rather than biological) explanation is true, we can expect a substantial reduction in (but not necessarily total abolition of) in-stent restenosis with drug eluting stents.
The impact of a device which promises to reduce restenosis (relative to a conventional stent) more than the Palmaz-Schatz stent did (relative to a balloon) can, therefore, be easily imagined. It is a situation analogous to that which developed in the mid 1990s, when use of the Palmaz-Schatz stent reduced angiographic restenosis from $32 \%$ after balloon angioplasty to $22 \%$ in the stent group, and the re-PCI rate from $21 \%$ to $10 \%$ (in highly selected lesions). ${ }^{5}$ As a result, interventional cardiology moved into the "stent era", stents being implanted in $70-90 \%$ of PCI procedures (in decidedly unselected lesions). It is natural that interventionists will want coated stents, and patients will demand them. This enthusiasm, however, comes with a cost. The Cypher drug-eluting stent is being marketed in the UK at a price about fivefold that of a bare stent. This mandates a vigorous appraisal of the financial implications of its use, at a time of expansion in PCI, in an increasingly cost conscious health service.

\section{WHO GETS RESTENOSIS?}

It is often stated that it is not possible to predict which patients will undergo restenosis. It is, however, true that certain lesion (and, to a lesser extent, patient) characteristics are associated with higher restenosis rates. Multivessel stenting and diabetes are the two main patient related variables increasing the probability of restenosis. In the case of diabetes, the relative risk is about 1.3:1. ${ }^{2}$ The evidence for specific lesion locations having high restenosis rates is generally unconvincing (such as the proximal left anterior descending $\operatorname{artery}^{67}$ ), an exception being the saphenous vein graft. $^{8}$ Angiographic, lesion related variables are more important, notably the minimum lumen diameter (MLD) preprocedure, $^{67}$ the MLD post-procedure. ${ }^{679}$ the acute gain, ${ }^{8}$ the vessel size (reference diameter), ${ }^{679}{ }^{10}$ stent length, ${ }^{79^{11-13}}$ and the presence of multiple stents (probably a manifestation of the same phenomenon). ${ }^{9}{ }^{12}$ Intravascular ultrasound (IVUS) based studies support these observations and show that an ostial location, plaque burden (plaque area/arterial area) and final

Abbreviations: $C A B G$, coronary artery bypass grafting; IVUS, intravascular ultrasound; MLD, minimum lumen diameter; $\mathrm{PCl}$, percutaneous coronary intervention; RAVEL, randomized comparison of a sirolimus-eluting stent with a standard stent for coronary revascularization; SIRIUS, US multicenter, randomized, double-blind study of the sirolimus-eluting stent in de novo coronary lesions; TLR, target lesion revascularisation; TVF, target vessel failure 
lumen dimensions (MLD and lumen cross sectional area) are also important. ${ }^{14}$ Approximately half of patients with angiographic restenosis (diameter stenosis $>50 \%$ ) present with clinical relapse. ${ }^{5}$ These usually require repeat revascularisation, the majority by PCI. On the other hand, "real world" restenosis rates are likely to be higher than those found in randomised controlled trials. Up-to-date, local data are important. Examination by one author (JG) of the records of 219 PCIs performed in 2001 (an unselected series, including acute and chronic cases, and all types of lesion) revealed that a mean of 1.6 vessels per patient were treated with a mean of 1.1 stents per vessel. The clinical restenosis (TLR) rate for these patients was $10.0 \%$ (of which $8.6 \%$ were for restenosis and $1.4 \%$ re-restenosis), $96 \%$ being treated with re-PCI and $4 \%$ with coronary artery bypass grafting (CABG). This figure of $10 \%$ TLR is very similar to those seen in unselected, contemporary, published series from other centres and operators. $^{15}$

\section{POTENTIAL STRATEGIES FOR USING DRUG ELUTING STENTS}

On the one hand, we could simply implant a drug eluting stent in every patient. This would substantially reduce the incidence of restenosis (perhaps by about two thirds, if we believe SIRIUS), and require no effort at predicting the most likely lesions to restenose. Initially, however, this approach would result in an approximately fourfold increase in stent budget (even with a discount in purchasing the drug eluting stents). A more responsible approach might be to identify a subset of lesions at the highest risk of restenosis, and target these for implantation of a drug eluting stent. This might yield a useful (though less substantial) reduction in restenosis at considerably less cost. In this paper, we aim to create a practical system for establishing different levels of risk of restenosis, and examine the impact of implanting drug eluting stents in lesions at different levels of risk.

\section{SELECTING PRACTICAL RISK FACTORS FOR IN-STENT RESTENOSIS}

Of all the risk factors for restenosis discussed above, we considered to be most useful "real world", lesion based risk factors that were readily prospectively measurable in the majority of patients undergoing PCI. We therefore rejected IVUS based variables and confined ourselves to simple, practical, quickly assessed, angiographic, lesion based parameters. We focused on intended stent width ( $\approx$ vessel reference diameter) and length $(\approx 7 \mathrm{~mm}$ longer than the lesion). MLD preprocedure was rejected as being too difficult to assess "by eye", and MLD post-procedure was rejected because it is post hoc. We obtained lesion based restenosis data from the literature. ${ }^{679101213}$ Extrapolation and estimation were necessary (because some papers considered the impact of stent length, while others considered the impact of reference diameter, and each used different stents). The only patient based variable important enough to be included in the analysis was diabetes. The risk of restenosis associated with multi-lesion stenting was assumed to be the sum of the risk of each lesion. We also assumed reasonable (but not exceptional) standards of stent implantation (a residual stenosis in the range 10-15\% is found even in well conducted studies ${ }^{3}$ ). We also determined the number of stents of each width and length used in contemporary practice from our catheter laboratory records. This was important to establish which stent sizes contributed most to the restenosis "burden" to the hospital in terms of sheer numbers.

\section{RISK OF RESTENOSIS BY STENT SIZE}

We plotted the angiographic restenosis rate, predicted by the literature, for different stent diameters ( $\approx$ reference diameter, divided into 0.25 and $0.5 \mathrm{~mm}$ groups) and lengths ( $\approx$ lesion +
Table 1 Angiographic restenosis rates for different widths and lengths of bare stents

\begin{tabular}{lllllll}
\hline $\begin{array}{l}\text { Stent } \\
\text { length } \\
(\mathrm{mm})\end{array}$ & \multicolumn{6}{l}{ Stent calibre $(\mathrm{mm})$} \\
\cline { 2 - 7 } & 2.25 & 2.5 & 2.75 & 3 & 3.5 & 4 \\
\hline$<10$ & 15 & 11 & 7 & 5 & 3 & 2 \\
& $(0)$ & $(5.5)$ & $(0.9)$ & $(8.3)$ & $(6)$ & $(1.6)$ \\
$10-14$ & 24 & 18 & 11 & 8 & 4 & 3 \\
& $(0.2)$ & $(5.8)$ & $(1.4)$ & $(13.1)$ & $(9.7)$ & $(3.4)$ \\
$15-19$ & & 27 & 16 & 12 & 6 & 4 \\
& $(0.2)$ & $(2.3)$ & $(3.2)$ & $(10.8)$ & $(9.9)$ & $(3)$ \\
$20-24$ & & 32 & 19 & 14 & 8 & 5 \\
& $(0)$ & $(0)$ & $(0.2)$ & $(0.9)$ & $(1.2)$ & $(0)$ \\
$25-29$ & & 39 & 23 & 17 & 9 & 6 \\
& $(0)$ & $(0.9)$ & $(0)$ & $(4.1)$ & $(3.4)$ & $(1.4)$ \\
$30-34$ & & 48 & 27 & 21 & 11 & 8 \\
& $(0)$ & $(0)$ & $(0)$ & $(0.2)$ & $(0)$ & $(0)$ \\
$>35$ & & 54 & 33 & 24 & 13 & 9 \\
& $(0)$ & $(0)$ & $(0)$ & $(0.2)$ & $(0)$ & $(0.2)$ \\
& & & & & &
\end{tabular}

Data are derived from the literature, 679101213 with extrapolation and approximation wherever necessary, and assume a residual in-stent post-procedural stenosis of $10-15 \%$.

Numbers in parentheses indicate the percentage stents of each size used at the Northern General Hospital, Sheffield

The bold numbers represent stent sizes at $>15 \%$ risk of restenosis in the general patient population, and sufficiently commonly implanted, to be eligible for implantation of a drug eluting stent.

The italicised numbers represent two further stent sizes which, when implanted in diabetic patients (approximately $20 \%$ of the PCI population), cross the $15 \%$ threshold of restenosis risk.

Other thresholds (for example, $10 \%$ or $5 \%$ ) may be considered and can readily be used to establish which stent sizes are eligible for implantation of a drug eluting stent (see text).

$7 \mathrm{~mm}$, divided into $5 \mathrm{~mm}$ groups $)^{679}$ in table 1 . We made the assumptions that the percentage increase in restenosis risk with increasing stent length applies proportionately to all stent widths, and that the percentage increase with decreasing stent width applies proportionately to all stent lengths. The per cent of each size of stent used in our hospital, broken down by the same criteria of length and width, was also plotted on this table. Table 1 reveals that stents of smaller calibre and longer length are at greater risk of restenosis-for example, a $2.5 \times 20 \mathrm{~mm}$ stent would be expected to have an angiographic restenosis rate of about $32 \%$. Significantly, it also shows how rare restenosis is in stents which are short and large in calibre-for example, a $4 \times 8 \mathrm{~mm}$ stent would be expected to have an angiographic restenosis rate of about $2 \%$. Table 1 also shows that there are relatively few stent sizes with a high risk of restenosis which are frequently used. When using table 1 it is important to remember that the figures quoted are merely a guide to the expected chance of angiographic restenosis. For the average patient ( $>1$ lesion with $>1$ stent) the composite angiographic restenosis rate is the sum of that of all the stents deployed and the clinical restenosis rate is about half of this composite figure.

\section{APPLICATION OF DIFFERENT RESTENOSIS THRESHOLDS}

Application of three arbitrary thresholds of lesion based restenosis risk ( $>5 \%,>10 \%$, and $>15 \%)$ to table 1 identified stent sizes with the highest priority for implantation of a drug eluting stent. For a 15\% restenosis threshold, few groups of stent sizes were found that were actually used (the others either had a low restenosis rate (large stent widths) or were hardly ever used (long stents with small widths)). The potentially important sizes, for this threshold, were $2.5 \times 10$ 
Table 2 Sizes of Cypher drug eluting stent marketed by Cordis

\begin{tabular}{llllllll}
\hline $\begin{array}{llllll}\text { Stent } \\
\text { length } \\
(\mathrm{mm})\end{array}$ & \multicolumn{2}{l}{ Stent calibre $(\mathrm{mm})$} \\
\cline { 2 - 7 } & 2.25 & 2.5 & 2.75 & 3 & 3.5 & 4 \\
\hline 8 & $*$ & $\dagger$ & $\dagger$ & $\dagger$ & $\dagger$ & $\dagger$ \\
13 & $*$ & $5.8(32)$ & $\dagger$ & $\dagger$ & $\dagger$ & $\dagger$ \\
18 & $*$ & $2.3(13)$ & $3.2(18)$ & $2.2(12)$ & $\dagger$ & $\dagger$ \\
23 & $*$ & $*$ & $0.2(1)$ & $0.2(1)$ & $\dagger$ & $\dagger$ \\
28 & $*$ & $*$ & $*$ & $4.1(23)$ & $* \dagger$ & $* \dagger$ \\
33 & $*$ & $*$ & $*$ & $*$ & $* \dagger$ & $* \dagger$ \\
\hline
\end{tabular}

Bold figures indicate stent sizes which should be considered for implantation in place of bare stents if the desired restenosis threshold is $15 \%$. As can be seen, these only comprise seven sizes, of which two $(3 \times 18 \mathrm{~mm}$ and $3 \times 23 \mathrm{~mm})$ are required only for diabetics. The figures outside the parentheses are the per cent of drug eluting stents as a proportion of all stents used (total $=18 \%$ for a $15 \%$ restenosis threshold). The figures in parentheses are the per cent of all the Cypher stents (total $=100 \%$ ).

*Insufficient stents of this size are implanted to make it worthwhile obtaining this size of Cypher.

tRestenosis rate $<15 \%$, so no Cypher is necessary. Equivalent numbers for any desired restenosis threshold (for example, $10 \%$ or $5 \%$ ) can be constructed by the reader from table 1 .

$20 \mathrm{~mm}, \quad 2.75 \times 15-25 \mathrm{~mm}$, and $3.0 \times 25-30 \mathrm{~mm}$ (plus $3.0 \times 15-25 \mathrm{~mm}$ for diabetics, who comprise about $20 \%$ of the PCI population). From the manufacturer's list of available sizes, we would require $2.5 \times 13,2.5 \times 18,2.75 \times 18,2.75 \times 23$, $3.0 \times 18,3.0 \times 23$, and $3.0 \times 28 \mathrm{~mm}$ sized Cypher stents. The numbers of these required per annum, expressed as percentage of the entire stock of stents, would be $18 \%$, distributed in the ratio $21: 8: 12: 1: 40: 3: 15$, respectively (table 2 ). For the $10 \%$ threshold, using tables 1 and 2 in a similar way, the proportion of drug eluting stents would be about $38 \%$ and, for the $5 \%$ threshold, about $77 \%$.

\section{IMPLICATIONS OF A POLICY OF SELECTIVE DRUG-ELUTING STENT IMPLANTATION UPON RESTENOSIS}

Multiplication of the per cent restenosis risk for each stent size category by the per cent of stents used in each size category (that is, the two figures in each box in table 1) yields the "restenosis burden" of each stent size. For example, the greatest restenosis burden $(130 / 903=14 \%)$ is provided by stents in the category $3.0 \times 15-20$, because they are numerous and have a moderate restenosis rate; whereas $2.5 \times 25-30 \mathrm{~mm}$ stents have a low restenosis burden (4\%) because, despite a high restenosis rate, they are rarely used. Addition of the figures for restenosis burden, for all stent sizes, reveals an overall, stent based, restenosis rate of $9.0 \%$. This stent based, angiographic restenosis rate, derived from independent data, is consistent with our own, "real world", clinical restenosis rate of 10\% (for 1.6 vessels per patient and 1.1 stents per vessel). Assuming that the stent sizes selected for drug eluting stent implantation have a restenosis rate about one third that of conventional stents (see first paragraph above), we can examine the impact upon restenosis of implanting drug eluting stents at selected thresholds of expected restenosis (first part of table 3). Application of a $15 \%$ restenosis risk threshold would result in reduction of this overall restenosis rate to $6.9 \%-\mathrm{a} 23 \%$ relative reduction. For the $10 \%$ threshold, the overall restenosis rate would fall to $5.4 \%$ - a $40 \%$ relative reduction. For the $5 \%$ threshold, the overall restenosis rate would fall to $3.7 \%-a$ $59 \%$ relative reduction. It would be reasonable to expect proportionately similar reductions in the patient based restenosis rate (from $10 \%$ to $7.7 \%, 6.0 \%$ or $4.1 \%$, respectively). Table 3 clearly shows that policies invoking progressively higher rates of implantation of a drug eluting stent exhibit a law of "diminishing returns" in terms of reduction in restenosis.

\section{WHAT WOULD BE THE COST OF A SELECTIVE STRATEGY?}

Stent prices vary from centre to centre and from country to country. Making some reasonable assumptions, and knowing the price of the Cypher quoted to us by Cordis, together with their discount levels for increasing numbers of stents ordered, however, allows us to construct some realistic estimates of the economic impact of various thresholds of drug eluting stent implantation (second part of table 3). Application of a restenosis risk threshold of $15 \%$, requiring $18 \%$ drug eluting stents, at the sizes listed above (with a 19\% price discount) would require an increase in the stent budget of about $55 \%$. A restenosis threshold of $10 \%$ (with a $22.5 \%$ discount) would cost an extra $110 \%$; and a threshold of 5\% (with a $22.5 \%$ discount) an extra $224 \%$. Policies invoking progressively higher rates of implantation of a drug eluting stent would, therefore, demand steep increases in total stent costs. On the other hand, a reduction in restenosis (and re-restenosis) would reduce the number of repeat PCIs. Notionally, this would result in attenuation of the extra cost associated with using the new stents (also shown in table 3). However, in the "real world", where expenditure is measured by the number of cases performed per year (not the number of patients), this would be less apparent, because patients with restenotic lesions would be replaced by those with de novo lesions. In these calculations, the number of other complications (CABG, myocardial infarction), which can add significantly to the costs created by an individual patient, are assumed to be few in number and equally distributed between conventional and drug eluting stents. ${ }^{4}$

\section{IS THIS THE BEST WAY TO SPEND EXTRA MONEY?}

Would the increase in stent expenditure resulting from the proposed use of a drug eluting stent be better spent on

Table 3 Sample figures for selection of drug eluting stents at different thresholds of restenosis risk

\begin{tabular}{|c|c|c|c|c|c|c|}
\hline $\begin{array}{l}\text { Restenosis } \\
\text { threshold (\%) }\end{array}$ & $\begin{array}{l}\text { Drug eluting stent to } \\
\text { be implanted (\%) }\end{array}$ & $\begin{array}{l}\text { New restenosis } \\
\text { rate of } \mathrm{PCl} \\
\text { population }\end{array}$ & $\begin{array}{l}\text { Lesions "saved" } \\
\text { from restenosis (\%) }\end{array}$ & $\begin{array}{l}\text { Increase in stent } \\
\text { budget* (a) (\%) }^{*} \text { ( }\end{array}$ & $\begin{array}{l}\text { Saving due to reduction } \\
\text { in restenosis (as \% stent } \\
\text { budgett) (b) }\end{array}$ & $\begin{array}{l}\text { Increase in total } \\
\text { budget (a-b) as \% } \\
\text { stent budget }\end{array}$ \\
\hline N/A (none) & 0 & 9 & 0 & 0 & 0 & 0 \\
\hline 15 & 18 & 6.9 & 2.1 & 55 & 11 & 44 \\
\hline 10 & 38 & 5.4 & 3.6 & 110 & 19 & 91 \\
\hline 5 & 77 & 3.7 & 5.2 & 224 & 28 & 196 \\
\hline 0 (all) & 100 & 3 & 6 & 288 & 32 & 256 \\
\hline
\end{tabular}

For example, if all lesions were stented with the new stent (bottom row), the institutional stent budget would increase by about $288 \%$, but because the restenosis rate would be reduced by about $2 / 3,6 \%$ lesions (approximately $6.7 \%$ patients) per year would be saved from restenosis, the cost of retreating whom amounts to $32 \%$ of the stent budget. The "net" cost increase with this policy is therefore $288-32=256 \%$ of the current stent budget; in practice, however, the $32 \%$ saving would not be seen because procedures for restenosis would be replaced by PCls performed on patients with de novo disease. *Assumes discounts of $12.5 \%$ for 1-200 drug eluting stents ordered per year, $19 \%$ for $200-400$, and $22.5 \%$ for $>400$.

$\dagger A^{\prime \prime \prime}$ notional" budgetary saving, because patients"'rescued" from PCl for restenosis will be replaced by new patients". 
increasing the number of (bare stent) PCIs? For a centre performing 700 PCIs per annum, current practice, with a $10 \%$ clinical restenosis rate, allows 630 patients to be treated, the remainder of the procedures being repeat PCIs for restenosis. Use of the Cypher stent in lesions with $>15 \%$ risk of restenosis, at a cost of an extra 55\% in stent expenditure, with a reduction in the overall clinical restenosis rate from $10 \%$ to $7.7 \%$, would increase this number to about 646; a gain of 16 patients. The same money could, instead, purchase approximately an extra 72 PCIs with conventional stents which, with a "conventional" restenosis rate of $10 \%$, amounts to 63 patients. This highlights nicely the dilemma; to balance moderately increased "quality" (clinical restenosis reduced from $10 \%$ to $7.7 \%$ ) and slightly increased "quantity" (an extra $2.5 \%$ patients treated in a year) against the possibility of no change in quality but considerably better quantity (an extra 10\% patients treated in a year). The greater the proposed expenditure on the drug eluting stents, the greater this difference; a few more cases of restenosis being saved, in contrast to the potential for many more de novo patients treated. In a financially constrained world, one could argue that the latter is preferable, provided that re-PCI is performed at low risk.

\section{MULTI-VESSEL PCI}

What will be the impact of using drug eluting stents in multivessel disease? The main reason for not performing multivessel PCI is no longer safety but restenosis. The main difference between multi-vessel stenting and CABG is the increased incidence of repeat revascularisation seen in the stent group. ${ }^{16}$ In simple terms, the patient based restenosis rate in such circumstances is the sum of the restenosis rate of each lesion treated. A "low restenosis" stent might, therefore, tip the balance in favour of treating multi-vessel disease with PCI rather than CABG in terms of clinical outcome. However, should all three lesions (or more) be treated with drug eluting stents (at huge expense)? Or should we adhere to our principles of "lesion based" restenosis risk? Our practice will have to be the latter, at least until costs come down. That approach also has an intrinsic "fairness"- every patient's restenosis risk will be proportionately reduced, whether from $21 \%$ to $7 \%$ in the case of a single lesion procedure at high risk of restenosis, or from $33 \%$ to $19 \%$ in the case of a triple vessel procedure including one high and two low risk ones. On the other hand, apart from the cost of a drug eluting stent, a PCI procedure is cheaper than a CABG procedure, so some attenuation of costs for the institution may be possible if more patients are treated with PCI rather than CABG.

\section{LIMITATIONS OF THIS PAPER}

The data in regard to the restenosis rates quoted in table 1 are, of necessity, based upon studies performed in the mid 1990s, mostly using the Palmaz-Schatz stent and dated implantation techniques and periprocedural treatment. Nevertheless, the figures appear to be consistent with our recent, "real world", patient based restenosis rates. Also, because no single data source contains a full spectrum of stent widths and lengths, we have had to extrapolate to construct table 1 (for example, in assuming that restenosis rates increase with stent length in the same proportion for different stent widths). It will also be appreciated that the data are derived from quantitative angiography rather than "eyeball" measurements (which most interventionists use). Equally, data from meticulously performed studies in the best centres may not apply so precisely to non-ideal lesions treated (in non-ideal ways) in everyday practice. We have, for instance, had to allow for the likelihood that the mean post-stent MLD is $10-15 \%$ (rather than $0 \%$ ). Finally, the costs associated with drug eluting stents are likely to fall. When they do, their use will broaden, although the principles underlying the analysis we have employed in this paper will remain valid.

\section{A DRUG ELUTING STENT FOR EVERY LESION?}

The RAVEL study included only small numbers of certain important lesion subsets; only $18 \%$ of vessels were $2.5 \mathrm{~mm}$, and mean lesion length was (a rather short) $9.6 \mathrm{~mm}$. There were no implantations in left main stems, bypass grafts, chronic total arterial occlusions, ostial stenoses, acute myocardial infarctions, in-stent restenoses, and bifurcations. Similar observations apply to the SIRIUS study. A "low restenosis" assumption for lesions other than the simplest may not be valid, and discretion should, therefore, be employed when considering drug eluting stent implantation in these contexts until appropriate studies have been completed. Furthermore, the Bx Velocity stent platform, upon which the Cypher stent is based, may not be suitable in certain situations-notably in tortuous vessels. It may also be the case that the development of stents eluting alternative therapeutic agents may be more appropriate in selected clinical settings. The data from these first studies with the sirolimus eluting stent are, however, so compelling that we are likely to see drug eluting stent implantation in lesions for which there is, as yet, little or no evidence for their utility (when the price comes down).

\section{SUMMARY}

A policy of selective implantation of drug-eluting stents, in a minority of lesions most likely to benefit, seems to be a rational way to employ this new technology. If the price of the device remains as high as presently planned, however, there is a strong argument that even this extra revenue might be better spent offering PCI with conventional stents to a considerably larger number of patients. In our hospital, we are unlikely to receive sufficient extra funds to implement anything other than a highly selective implantation policy, probably employing the $15 \%$ restenosis threshold, as described in this paper. We will then audit our results to ensure that a real reduction in restenosis is achieved. We also plan to review the restenosis "threshold" for choosing a drug eluting stent that seems economically sustainable, using the data presented here, in the light of any changes in the price of the product, the savings obtained in treating restenosis, and the emergence of competitor devices.

\section{Authors' affiliations}

J Gunn, A C Morton, C Wales, C M H Newman, D C Crossman, Cardiovascular Research Group, Division of Clinical Sciences (Northern General Hospital), University of Sheffield, Sheffield, UK

D C Cumberland, Ampang Puteri Specialist Hospital, Jalang Memanda, Mukin Ampang, Selangor, Malaysia

\section{REFERENCES}

1 Morice MC, Serruys PW, Sousa JE, et al. A randomised comparison of a sirolimus-eluting stent with a standard stent for coronary revascularization. N Engl J Med 2002;346:1773-80.

2 Sousa JE, Costa MA, Abizaid AC, et al. Sustained suppression of neointimal proliferation by sirolimus-eluting stents. Circulation 2001;104:2007-1 1.

3 Marx SO, Marks AR. The development of rapamycin and its application to stent restenosis. Circulation 2001;104:852-5.

4 SIRIUS Trial. Summary of the results of the first 400 patients. Data obtainable form: Cordis (Johnson and Johnson), Coronation Rd, South Ascot, SL5 9EY, UK; cordis@medgb.jnj.com

5 Serruys PW, de Jaegere P, Kiemeneij F, et al. A comparison of balloon-expandable-stent implantation with balloon angioplasty in patients with coronary artery disease. N Engl J Med 1994;331:489-95

6 Serruys PW, Kay P, Disco C, et al. Periprocedural quantitative coronary angiography after Palmaz-Schatz stent implantation predicts the restenosis rate at six months. J Am Coll Cardiol 1999;34:1067-74.

7 Kobayashi Y, De Gregorio J, Kobayashi N, et al. Stented segment length as an independent predictor of restenosis. J Am Coll Cardiol 1999;34:651-9.

8 Heidland UE, Heintzen MP, Michel CJ, et al. Risk factors for the development of restenosis following stent implantation of venous bypass grafts. Heart 2001;85:312-7.

9 Kornowski R, Bharghava B, Fuchs S, et al. Procedural results and late clinical outcomes after percutaneous interventions using long $(\geqslant 25 \mathrm{~mm}$ versus short $(<20 \mathrm{~mm})$ stents. J Am Coll Cardiol 2000;35:612-8.

10 Bauters C, Hubert E, Prat A et al. Predictors of restenosis after coronary stent implantation. J Am Coll Cardiol 1998;31:1291-8. 
11 Heidland $U$, Heintzen M, Michel $C$ et al. Risk factors for the development of restenosis following stent implantation of venous bypass grafts. Heart 2001;85:312-7.

12 Kastrati A, Elezi S, Dirschinger J, et al. Influence of lesion length on restenosis after coronary stent placement. Am J Cardio 1999:83:1617-22

13 Mercado N, Boersma E, Wijns W et al. Clinical and quantitative coronary angiographic predictors of coronary restenosis. J Am Coll Cardiol 2001; $\mathbf{3 8 : 6 4 5 - 5 2 . ~}$
14 Hoffmann R, Mintz GS, Mehran R, et al. Intravascular ultrasound predictors of angiographic restenosis in lesions treated with Palmaz-Schatz stents. J Am Coll Cardiol 1998;31:43-9.

15 Elbaz M, El Mokhtar E, Fourcade J, et al. Does stent design affect the long-term outcome after coronary stenting? Cathet Cardiovasc Intervent 2002:56:305-11

16 Serruys $\mathbf{P}$, Unger $F$, Sousa J, et al. Comparison of coronary artery bypass surgery and stenting for the treatment of multivessel disease. N Engl J Med 2001:344:11117-24.

\section{IMAGES IN CARDIOLOGY}

\section{A giant thrombus aspirated from a coronary artery}

72 year old woman was admitted to the emergency department with persistent chest pain. The ECG showed ST segment elevations in the inferior leads. She was diagnosed with acute myocardial infarction. Primary percutaneous coronary intervention (PCI) was performed. At first balloon angioplasty was applied. Coronary artery angiography revealed tandem defects in the right coronary artery after the procedure. We considered that the defect indicated a thrombus and attempted to aspirate it using the Rescue percutaneous thrombectomy system (Rescue PT, Boston Scientific Corp, Maple Grove, Minnesota, USA). The Rescue PT system consists of a flexible, dual lumen Monorail 4.5 French catheter and a collection bottle with a filter for separating solid bodies from blood that contains small pieces of thrombus and atheromatous plaque. A catheter and a vacuum assistance are used to break clots into small pieces that are collected into a bottle. This catheter was first inserted past the lesion and then pulled back slowly. A giant thrombus was withdrawn while
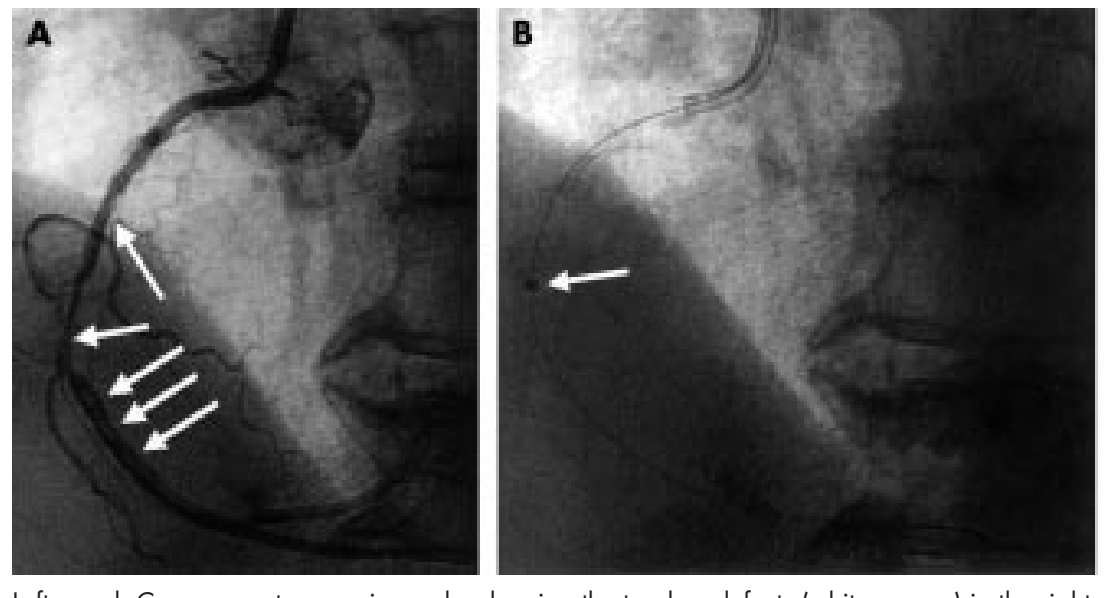

attached to the tip of the aspiration catheter through the PCI guide catheter, without entering the usual aspiration hole of the Rescue catheter from the coronary artery.

Coronary artery angiography showed the defects decreased after aspiration therapy. Thus PCI was easily successful with adjunctive stenting. When there is a large thrombus in the coronary artery, it is very useful to aspirate it. We conclude that it is necessary to undergo this pullback procedure after aspiration so that blood containing thrombus in the PCI guide catheter is withdrawn fully, to avoid the thrombus remaining in the PCI guide catheter.

T Miyamoto

T Sasaki

A Niwa miyamoto@musashino.jrc.or.jp

Left panel: Coronary artery angiography showing the tandem defects (white arrows) in the right coronary artery before aspiration therapy. Middle panel: Undertaking aspiration therapy. The $\mathrm{PCl}$ guide wire reaches the distal branch in the coronary artery. The white arrow indicates the tip of the aspiration catheter. Right panel: After aspiration therapy, the defects nearly disappear.

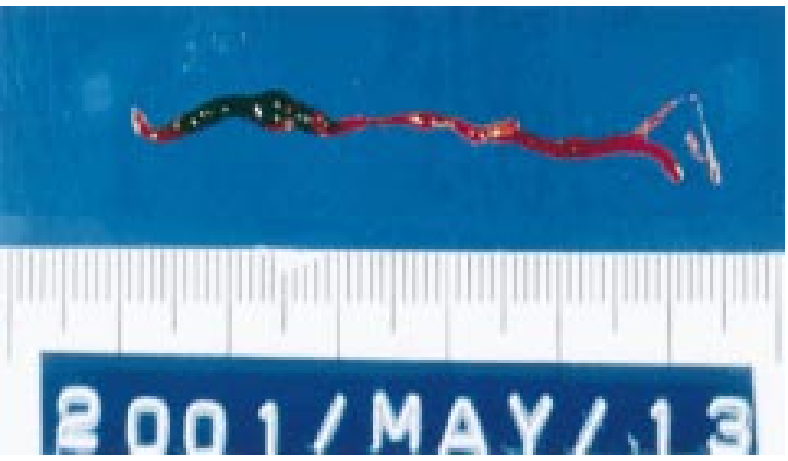

Aspirated giant thrombus, nearly $5.5 \mathrm{~cm}$ long.

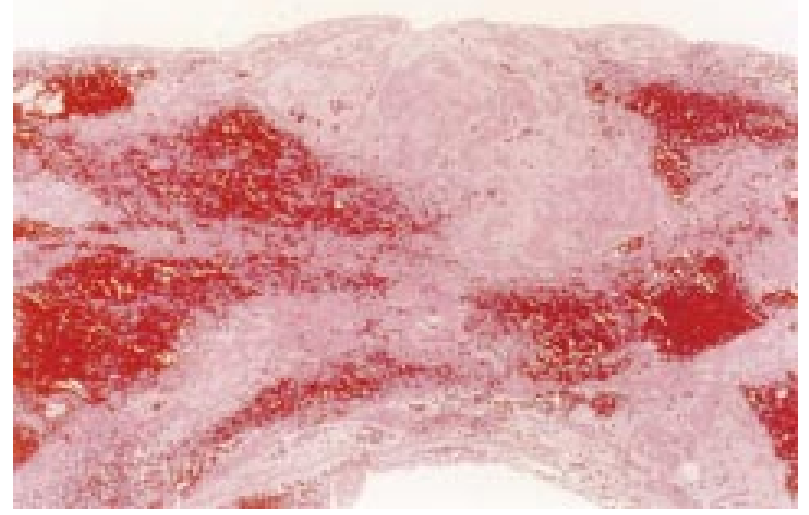

Haematoxylin and eosin staining of the aspirated giant thrombus. The material comprises mostly fibrin complexes and red and white blood cells. 